\title{
Correction to: Conformer Analysis of a Biological G-Quadruplex Element in the Human c-MYC Promoter by Native Polyacrylamide Gel Electrophoresis
}

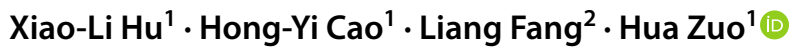

Published online: 1 July 2021

(C) The Nonferrous Metals Society of China 2021

\section{Correction to: \\ Journal of Analysis and Testing (2021) 5:188-194 \\ https://doi.org/10.1007/s41664-021-00181-0}

In the original publication of the article, the first author's first name has been incorrectly published. The correct author name is Xiao-Li Hu.

The original article was corrected.

Liang Fang

fangliangkkk@163.com

$\triangle$ Hua Zuo

zuohua@swu.edu.cn

1 Key Laboratory of Luminescence Analysis and Molecular Sensing (Southwest University), Ministry of Education, College of Pharmaceutical Sciences, Southwest University, Chongqing 400715, China

2 Department of Oncology, The Ninth People's Hospital of Chongqing, Chongqing 400700, China 